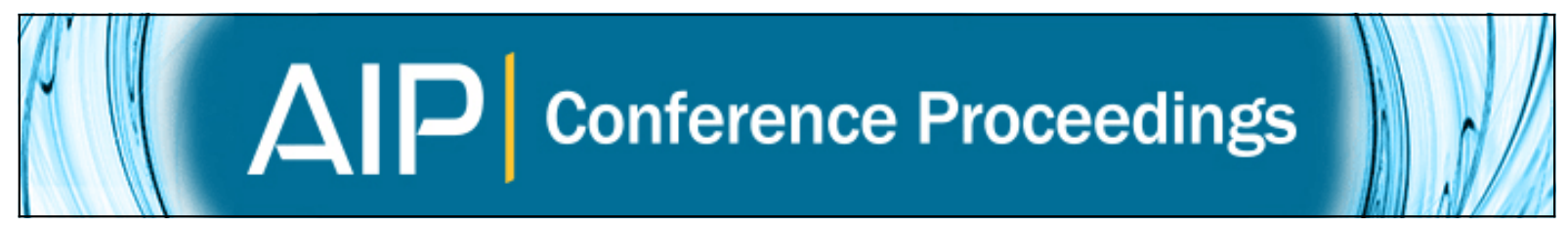

\title{
Slowing and stopping light with an optomechanical crystal array
}

D. E. Chang, A. H. Safavi-Naeini, M. Hafezi, and O. Painter

Citation: AIP Conference Proceedings 1291, 13 (2010); doi: 10.1063/1.3506102

View online: http://dx.doi.org/10.1063/1.3506102

View Table of Contents: http://scitation.aip.org/content/aip/proceeding/aipcp/1291?ver=pdfcov

Published by the AIP Publishing

Articles you may be interested in

Coupling strategies for coherent operation of quantum cascade ring laser arrays

AIP Conf. Proc. 1399, 989 (2011); 10.1063/1.3666710

Experimental demonstration of the slow group velocity of light in two-dimensional coupled photonic crystal microcavity arrays

Appl. Phys. Lett. 86, 111102 (2005); 10.1063/1.1882755

Guiding $1.5 \mu \mathrm{m}$ light in photonic crystals based on dielectric rods

Appl. Phys. Lett. 85, 6110 (2004); 10.1063/1.1840107

Observation of light propagation in two-dimensional photonic crystal-based bent optical waveguides

J. Appl. Phys. 89, 855 (2001); 10.1063/1.1336517

Propagation of light beams along line defects formed in a- Si/SiO 2 three-dimensional photonic crystals:

Fabrication and observation

Appl. Phys. Lett. 74, 777 (1999); 10.1063/1.123364 


\title{
Slowing and stopping light with an optomechanical crystal array
}

\author{
D.E. Chang* ${ }^{*}$ A.H. Safavi-Naeini ${ }^{\dagger}$, M. Hafezi** and O. Painter ${ }^{\dagger}$ \\ * Institute for Quantum Information, California Institute of Technology, Pasadena, CA 91125 \\ ${ }^{\dagger}$ Thomas J. Watson, Sr., Laboratory of Applied Physics, California Institute of Technology, Pasadena, CA 91125 \\ ${ }^{* *}$ Joint Quantum Institute and Department of Physics, University of Maryland, College Park, MD 20742
}

\begin{abstract}
The ability to coherently store and retrieve optical information in a rapidly tunable manner is an important ingredient for all-optical information processing. In the classical domain, this optical buffering is necessary to manage information flow in complex networks. In quantum information processing, such a system can also serve as a long-term memory capable of storing the full quantum information contained in an optical pulse. Here we suggest a novel approach to light storage involving an optical waveguide coupled to an optomechanical crystal array, where light in the waveguide can be dynamically and reversibly mapped into long-lived mechanical vibrations in the array. This technique enables large bandwidths and long storage and delay times in a compact, on-chip platform.
\end{abstract}

Keywords: optomechanics, nanomechanics, slow light

PACS: $07.10 . \mathrm{Cm}, 42.50 . \mathrm{Wk}, 42.50 . \mathrm{Ex}$

\section{INTRODUCTION}

A number of schemes to coherently delay and store optical information are being actively explored. These range from tunable coupled resonator optical waveguide (CROW) structures [1, 2], where the propagation of light is dynamically altered by modulating the refractive index of the system, to electromagnetically induced transparency (EIT) in atomic media [3, 4], where the optical pulse is reversibly mapped into internal atomic degrees of freedom. While these schemes have been demonstrated in a number of remarkable experiments [5, $6,7,8]$, they remain difficult to implement in a practical setting.

Here, we present a novel approach to store or stop an optical pulse propagating through a waveguide, wherein coupling between the waveguide and a nearby nanomechanical resonator array enables one to map the optical field into long-lived mechanical excitations. Our scheme combines many of the best attributes of previously proposed approaches, in that it simultaneously allows for large bandwidths of operation, on-chip integration, relatively long delay/storage times, and ease of external control. The possibility of observing quantum behavior in nano- and opto-mechanical systems has attracted considerable interest in recent years [9]. Beyond fundamental interest, our present work opens up the possibility of a novel major application for such systems quantum or classical all-optical information processing.

\section{DESCRIPTION OF SYSTEM: AN OPTOMECHANICAL CRYSTAL ARRAY}

An optomechanical crystal [10] is a periodic structure that constitutes both a photonic [11] and a phononic [12] crystal. The ability to engineer optical and mechanical properties in the same structure should enable unprecedented control over light-matter interactions. Planar twodimensional (2D) photonic crystals, formed from patterned thin dielectric films on the surface of a microchip, have been succesfully employed as nanoscale optical circuits capable of efficiently routing, diffracting, and trapping light. Fabrication techniques for such 2D photonic crystals have matured significantly over the last decade, with experiments on a Si chip [13] demonstrating excellent optical transmission through long $(N>100)$ linear arrays of coupled photonic crystal cavities. In a similar Si chip platform it has recently been shown that suitably designed photonic crystal cavities also contain localized acoustic resonances which are strongly coupled to the optical field via radiation pressure [10]. These planar optomechanical crystals (OMCs) are thus a natural candidate for implementation of our proposed slow-light scheme, although the phenomenon is quite general to any array of optomechanical systems.

In the following we consider an optomechanical crystal containing a periodic array of such defect cavities (see Fig. 1a). Each element of the array contains two optical cavity modes (denoted 1,2) and a co-localized mechanical resonance (denoted $m$ ). The modes 2 are classically pumped, and the frequencies are chosen such that $\omega_{1}=\omega_{2}+\omega_{m}$. The pump photons facilitate Raman scat-

CP1291, The Third International Workshop on Theoretical and Computational Nanophotonics (TaCoNa-Photonics 2010) edited by D. M. Chigrin

(C) 2010 American Institute of Physics 978-0-7354-0846-3/10/\$30.00 
a)

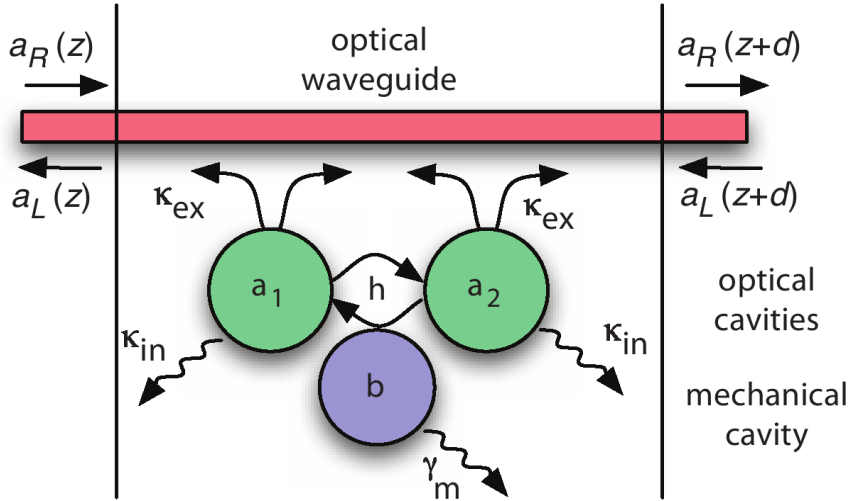

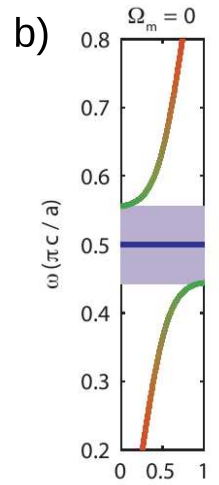
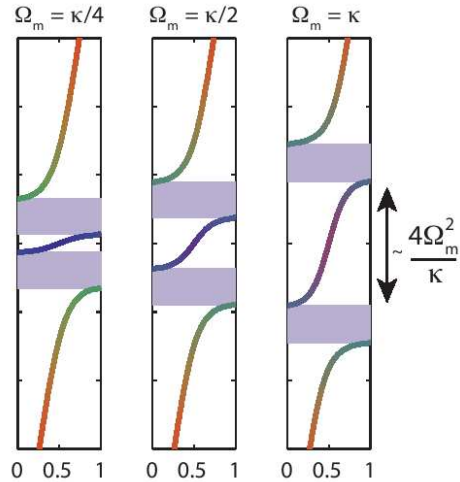

$\mathrm{K}(\pi / \mathrm{d})$
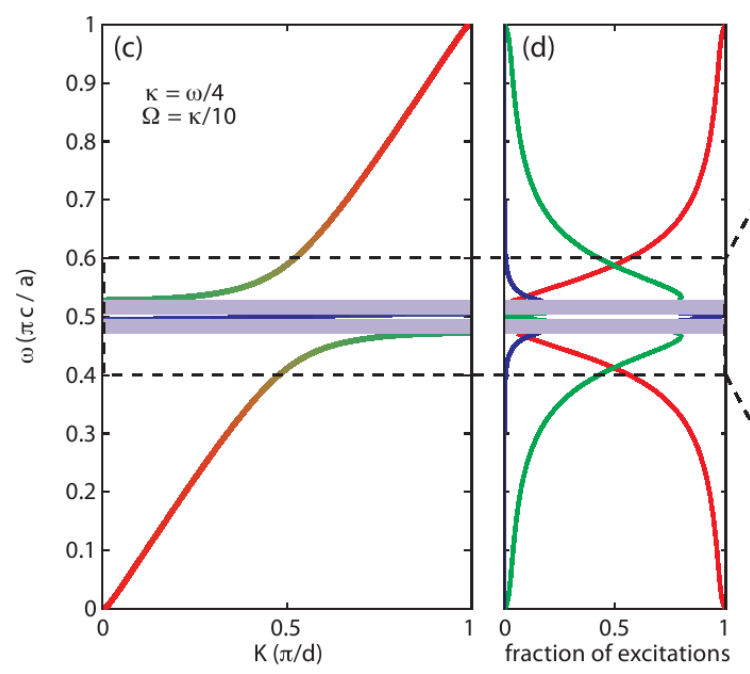

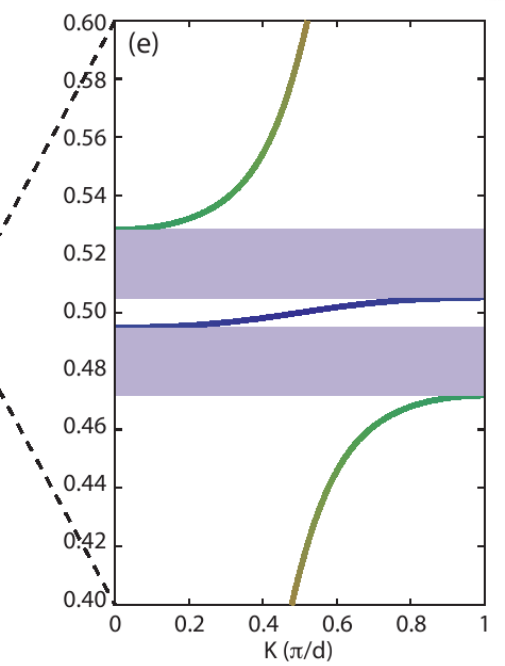

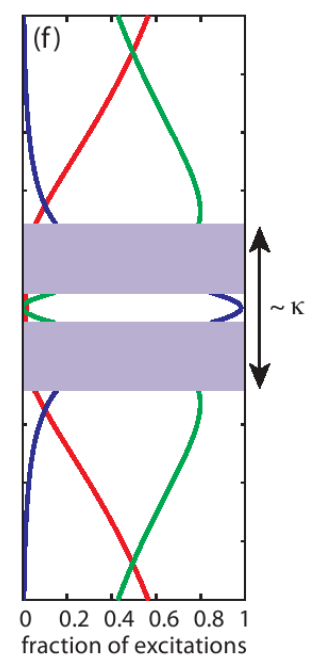

FIGURE 1. a) Illustration of a double optical cavity system forming the unit cell of the optomechanical array. A two-way optical waveguide is coupled to a pair of optical cavity modes $a_{1}$ and $a_{2}$, whose resonance frequencies differ by the frequency of the mechanical mode $b$. Both optical modes leak energy into the waveguide at a rate $\kappa_{\text {ex }}$ and have an inherent decay rate $\kappa_{\text {in }}$. The mechanical resonator optomechanically couples the two optical resonances with a cross coupling rate of $h$. b) The band structure of the system, for a range of driving strengths between $\Omega_{m}=0$ and $\Omega_{m}=\kappa$. The blue shaded regions indicate band gaps, while the color of the bands elucidates the fractional occupation (red for energy in the optical waveguide, green for the optical cavity, and blue for mechanical excitations). The dynamic compression of the bandwidth is clearly visible as $\Omega_{m} \rightarrow 0$. c) Band structure for the case $\Omega_{m}=\kappa / 10$ is shown in greater detail. d) The fractional occupation for each band in c) is plotted separately. It can be seen that the polaritonic slow-light band is mostly mechanical in nature, with a small mixing with the waveguide modes and negligible mixing with the optical cavity mode. Zoom-ins of figures c) and d) are shown in e) and f).

tering events where a photon in mode 1 is destroyed and a phonon is created, or vice versa. The Rabi frequency $\Omega_{m}(t)=h \alpha_{2}(t)$ of this process depends on the intracavity field ampiltude of mode 2 (which is readily tunable) and the per-phonon cross-coupling strength $h$ between modes 1 and 2 . In addition, the cavity modes 1 are coupled to a common two-way waveguide. The tunable coupling between phonons and photons is the key mechanism to reversibly map propagating fields in the waveguide (through mode 1) to mechanical excitations along the array. This Raman scattering process is a wellknown feature of optomechanical systems. For instance, this mechanism has been suggested as a way to opti- cally cool the mechanical motion to its quantum ground state $[14,15,16,17,18,9]$. Here, we show that the optomechanical interaction not only facilitates cooling, but also yields a rapidly varying susceptibility for an incoming optical field in the waveguide, which in turn enables a tunable slow group velocity.

The design considerations for the optomechanical crystal are described in detail below. For now, we take as realistic parameters $\omega_{1} / 2 \pi=200 \mathrm{THz}$, $\omega_{m} / 2 \pi=10 \mathrm{GHz}, h / 2 \pi=0.35 \mathrm{MHz}$, and mechanical and (unloaded) optical quality factors of $Q_{m} \equiv \omega_{m} / \gamma_{m} \sim 10^{3}$ (room temperature)- $10^{5}$ (low temperature) and $Q_{1} \equiv \omega_{1} / \kappa_{1, \text { in }}=3 \times 10^{6}$, where $\gamma_{m}$ is the 
mechanical decay rate and $\kappa_{1 \text {,in }}$ is the intrinsic optical cavity decay rate. In practice, one can also over-couple cavity mode 1 to the waveguide, with a waveguideinduced optical decay rate $\kappa_{\mathrm{ex}}$ that is much larger than $\kappa_{\text {in. }}$

\section{SLOWING AND STOPPING LIGHT}

The propagation characteristics of light in the waveguide and interacting with the optomechanical crystal is characterized by an optical band structure, which is shown in Figs. 1b-f. The color coding of the dispersion curves (red for waveguide, green for optical cavity, blue for mechanical resonance) indicates the distribution of energy or fractional occupation in the various degrees of freedom of the system in steady-state. Far away from the cavity resonance, the dispersion relation is nearly linear and simply reflects the character of the input optical waveguide, while the propagation is strongly modified near resonance $\left(\omega=\omega_{1}=\omega_{2}+\omega_{m}\right)$. In the absence of optomechanical coupling $\left(\Omega_{m}=0\right)$, a transmission band gap of width $\sim \kappa$ forms around the optical cavity resonance (reflections from the bare optical cavity elements constructively interfere). In the presence of optomechanical driving, the band gap splits in two (blue shaded regions) and a new propagation band centered around the cavity resonance appears in the middle of the band gap. For weak driving $\left(\Omega_{m}<\kappa\right)$ the width of this band is $\sim 4 \Omega_{m}^{2} / \kappa$, while for strong driving $\left(\Omega_{m}>\kappa\right)$ one recovers the "normal mode splitting" of width $\sim 2 \Omega_{m}$ [19]. This relatively flat polaritonic band yields the slow-light propagation of interest. Indeed, for small $\Omega_{m}$ the steady-state energy in this band is almost completely mechanical in character, indicating the strong mixing and conversion of energy in the waveguide to mechanical excitations along the array.

The Bloch wavevector near resonance is given by

$$
k_{\mathrm{eff}} \approx k_{0}+\frac{\kappa_{\mathrm{ex}} \delta_{k}}{2 d \Omega_{m}^{2}}+\frac{i \kappa_{\mathrm{ex}} \kappa_{\mathrm{in}} \delta_{k}^{2}}{4 d \Omega_{m}^{4}}+\frac{\left(2 \kappa_{\mathrm{ex}}^{3}+12 \kappa_{\mathrm{ex}} \Omega_{m}^{2}\right) \delta_{k}^{3}}{24 d \Omega_{m}^{6}} .
$$

Here $k_{0}$ is the resonant wavevector, $d$ is the distance between elements, and $\delta_{k}$ is the frequency detuning from resonance. The group velocity through the coupled waveguide $v_{g}=\left(d k_{\text {eff }} / d \delta_{k}\right)^{-1}=2 d \Omega_{m}^{2} / \kappa_{\text {ex }}$ can be dramatically slowed by an amount that is tunable through the optomechanical coupling strength $\Omega_{m}$. The quadratic and cubic terms in $k_{\text {eff }}$ characterize pulse absorption and group velocity dispersion, respectively. For a system of $N$ elements, these processes yield a static bandwidth-delay product of

$$
\Delta \omega \tau_{\text {delay }} \sim \min \left(\sqrt{2 N \kappa_{\mathrm{ex}} / \kappa_{\mathrm{in}}},\left(6 \pi N^{2}\right)^{1 / 3}\right)
$$

for constant $\Omega_{m}$ and negligible mechanical losses. The first term on the right is the limit set by absorption, while the second term is the limit set by pulse distortion. When intrinsic optical cavity losses are negligible, and if one is not concerned with pulse distortion, one can propagate light over the full bandwidth $\sim 4 \Omega_{m}^{2} / \kappa$ of the slowlight polariton band and the bandwidth-delay product increases to $\Delta \omega \tau_{\text {delay }} \sim N$.

In the static regime, the scaling of bandwidth-delay product obtained here is identical to CROW systems [2]. In the case of EIT, a static bandwidth-delay product of $\Delta \omega \tau_{\text {delay }} \sim \sqrt{\text { OD }}$ results, where OD is the optical depth of the atomic medium. This product is limited by photon absorption and re-scattering into other directions, and is analogous to our result $\Delta \omega \tau_{\text {delay }} \sim \sqrt{N \kappa_{\text {ex }} / \kappa_{\text {in }}}$ in the case of large intrinsic cavity linewidth. On the other hand, when $\kappa_{\text {in }}$ is negligible, photons are never lost and reflections can be suppressed by interference. This yields an improved scaling $\Delta \omega \tau_{\text {delay }} \sim N^{2 / 3}$ or $\sim N$, depending on whether one is concerned with group velocity dispersion. In atomic media, the weak atom-photon coupling makes achieving OD $>100$ very challenging. In contrast, in our system as few as $N \sim 10$ elements would be equivalently dense.

While we have thus far shown the static dispersion curves, we now argue that the group velocity $v_{g}(t)=$ $2 d \Omega_{m}^{2}(t) / \kappa_{\text {ex }}$ can in fact be adiabatically changed once a pulse is completely localized inside the system, leading to distortion-less propagation at a dynamically tunable speed. In particular, by tuning $v_{g}(t) \rightarrow 0$, the pulse can be completely stopped and stored.

This phenomenon can be understood in terms of a "dynamic compression" of the pulse bandwidth. The same physics applies for CROW structures $[1,20]$, and the argument is re-summarized here. First, under constant $\Omega_{m}$, an optical pulse completely enters the medium within the bandwidth of the polariton band. Once the pulse is inside, we consider the effect of a gradual reduction in $\Omega_{m}(t)$. Decomposing the pulse into Bloch wavevector components, it is clear that each Bloch wavevector is conserved under arbitrary changes of $\Omega_{m}$ (as it is fixed by the system periodicity). Furthermore, transitions to other bands are negligible provided that the energy levels are varied adiabatically compared to the size of the gap. In this case, the bandwidth of the pulse is dynamically compressed, and the reduction in slope of the polariton band (Fig. 1) causes the pulse to propagate at an instantaneous group velocity $v_{g}(t)=2 d \Omega_{m}^{2}(t) / \kappa_{\mathrm{ex}}$ without any distortion. In the limit that $\Omega_{m} \rightarrow 0$, the polaritonic band becomes flat and completely mechanical in character, indicating that the pulse has been reversibly and coherently mapped onto stationary mechanical excitations within the array. 


\section{LIMITATIONS TO STORAGE}

Given that the polaritonic band is mostly mechanical for realistic systems, the maximum storage time is set by the mechanical decay rate, $\sim 1 / \gamma_{m}$. For parameters $\omega_{m} / 2 \pi=10 \mathrm{GHz}$ and $Q_{m}=10^{5}$, this yields a storage time of $\sim 10 \mu \mathrm{s}$, which is much longer than that of realistic CROW structures. The key feature of our system is that we effectively "down-convert" the high-frequency optical fields to low-frequency mechanical excitations, which naturally decay over much longer time scales. While storage times of $\sim 10 \mathrm{~ms}$ are possible using atomic media [21], their bandwidths so far have been limited to $<1 \mathrm{MHz}$ [20]. In our system, the width of the polariton band depends on the circulating intensity in the "tuning" cavities, but as an example, a width of $\sim 1 \mathrm{GHz}$ is possible using an intra-cavity average photon number of $\left|\alpha_{2}^{2}\right| \sim 10^{7}$.

One of the major sources of error in our device will be mechanical noise, which through the optomechanical coupling can be mapped into noise power in the optical waveguide output. This is essentially the price that one pays for down-converting optical excitations to mechanical to yield longer storage times - in turn, any mechanical noise gets "up-converted" to optical energy (whereas, for example, the probability of having a thermal optical photon is negligible). Defining an effective optically induced cooling rate $\Gamma_{\mathrm{opt}}=4 \Omega_{m}^{2} / \kappa$, it can be shown that when $\Gamma_{\text {opt }} \gg \gamma_{m}$ the optical noise power emerging from one end of the waveguide is given by

$$
P_{\text {noise }} \approx \frac{N \hbar \omega_{1}}{2} \frac{\kappa_{\mathrm{ex}}}{\kappa}\left(\gamma_{m} \bar{n}_{\mathrm{th}}+\Gamma_{\mathrm{opt}}\left(\frac{\kappa}{4 \omega_{m}}\right)^{2}\right) .
$$

The first term on the right corresponds to the upconversion of thermal mechanical noise $\left(\bar{n}_{\mathrm{th}}=\left(e^{\hbar \omega_{m} / k_{B} T_{b}}-\right.\right.$ $1)^{-1}$ is the Bose occupation number at the mechanical frequency and $T_{b}$ is the bath temperature), while the second term corresponds to optically-induced Stokes scattering (where a pump photon in cavity 2 creates both a photon in cavity 1 and a phonon). Generally, the thermal noise can be suppressed by working at lower temperatures, while Stokes scattering can be suppressed with good sideband resolution, $\kappa \ll \omega_{m}$.

At room temperature, $\bar{n}_{\mathrm{th}} \approx k_{B} T_{b} / \hbar \omega_{m}$ is large and thermal noise will dominate, yielding a noise power of $\sim 0.4 \mathrm{nW}$ per element for previously given system parameters and $\kappa_{\mathrm{ex}} / \kappa \approx 1$. We note that the thermal noise scales inversely with mechanical frequency, and the use of high-frequency mechanical oscillators ensures that such noise remains easily tolerable even at room temperature. At cryogenic temperatures, these high-frequency oscillators can be thermally cooled to the ground state, which enables our device to operate as a quantum memory for photons. A more detailed analysis shows that up to $\sim 100$ single-photon pulses may be stored in our system under realistic conditions.

\section{OPTOMECHANICAL CRYSTAL DESIGN}

A schematic showing a few periods of our proposed 2D OMC slow-light structure is given in Fig. 2. The structure is built around a "snowflake" crystal pattern of etched holes into a Silicon slab [22]. This pattern, when implemented with a physical lattice constant of $a=400 \mathrm{~nm}$, snowflake radius $r=168 \mathrm{~nm}$, and snowflake width $w=60 \mathrm{~nm}$ (see Fig. 2a), provides a simultaneous phononic bandgap from 8.6 to $12.6 \mathrm{GHz}$ and a photonic pseudo-bandgap from 180 to $230 \mathrm{THz}$. A single point defect, formed by removing two adjacent holes (a so-called "L2" defect), yields the co-localized phononic and photonic resonances shown in Figs. $2 b$ and $c$, respectively. The optomechanical coupling between the two resonances can be quantified by a coupling rate, $g$, which corresponds to the frequency shift in the optical resonance line introduced by a single phonon displacement. Numerical finite-element-method (FEM) simulations of the L2 defect indicate the mechanical resonance occurs at $\omega_{m} / 2 \pi=11.2 \mathrm{GHz}$, with a coupling rate of $g / 2 \pi=489 \mathrm{kHz}$ to the optical mode at frequency $\omega_{o} / 2 \pi=199 \mathrm{THz}$.

In order to form the double-cavity system described in the slow-light scheme above, a pair of L2 cavities are placed in the near-field of each other as shown in the dashed box region of Fig. 2a. Modes of the two degenerate L2 cavities mix, forming supermodes of the double-cavity system which are split in frequency. The frequency splitting between modes can be tuned via the number of snowflake periods between the cavities. The optomechanical cross-coupling of the odd $\left(\mathbf{E}_{-}\right)$and even $\left(\mathbf{E}_{+}\right)$optical supermodes mediated by the motion of the odd parity mechanical supermode $\left(\mathbf{Q}_{-}\right)$of the doublecavity drives the slow-light behavior of the system. We let cavity modes 1,2 in our system correspond to $\mathbf{E}_{-}$and $\mathbf{E}_{+}$, respectively. Since $\mathbf{Q}_{-}$is a displacement field that is antisymmetric about the two cavities, there is no optomechanical self-coupling between the optical supermodes and this mechanical mode. On the other hand, the crosscoupling between the two different parity optical supermodes is large and given by $h=g / \sqrt{2}=2 \pi(346 \mathrm{kHz})$.

The different spatial symmetries of the optical cavity supermodes allow them to be addressed independently. To achieve this we create a pair of linear defects in the snowflake lattice as shown in Fig. 2a, each acting as a single-mode optical waveguide at the desired frequency of roughly $200 \mathrm{THz}$. Sending light down both waveguides, with the individual waveguide modes either in or out of phase with each other, will then excite the even or odd supermode of the double cavity, respectively. The 


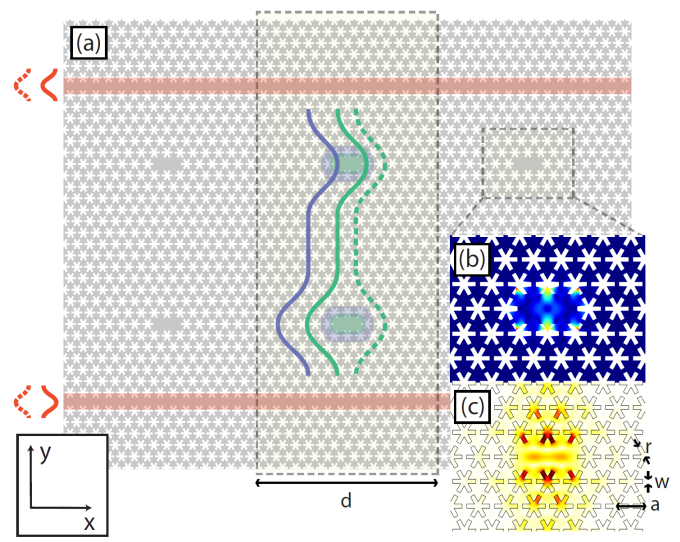

FIGURE 2. a) Top view of our proposed implementation of the optomechanical crystal array, with the superlattice unit-cell of length $d$ highlighted in the center. The displacement field amplitude $|\mathbf{Q}(\mathbf{r})|$ of the mechanical mode and in-plane electric field amplitude $|\mathbf{E}(\mathbf{r})|$ of the optical mode are shown in b) and c), respectively, for a single L2 defect cavity.

waveguide width and proximity to the $\mathrm{L} 2$ cavities can be used to tune the cavity loading, which for the structure in Fig. 2a results in the desired $\kappa_{\mathrm{ex}} / 2 \pi=2.4 \mathrm{GHz}$. It should be noted that these line-defect waveguides do not guide phonons at the frequency of $\mathbf{Q}_{-}$, and thus no additional phonon leakage is induced in the localized mechanical resonance.

\section{OUTLOOK}

The possibility of using optomechanical systems to facilitate major tasks in classical optical networks has been suggested in several recent proposals [23, 24]. This present work not only extends these prospects, but proposes a fundamentally new direction where optomechanical systems can be used to control and manipulate light at a quantum mechanical level. Such efforts would closely mirror proposals to perform similar tasks using EIT and atomic ensembles [4]. At the same time, the optomechanical array has a number of novel features compared to atoms, in that each element can be deterministically positioned, addressed, and manipulated, and a single element is already optically dense. Taken together, this raises the possibility that mechanical systems can provide a novel, highly configurable on-chip platform for realizing quantum optics and "atomic" physics.

\section{ACKNOWLEDGMENTS}

This work was supported by the DARPA/MTO ORCHID program through a grant from AFOSR. DC acknowl- edges support from the Gordon and Betty More Foundation through Caltech's Center for the Physics of Information. ASN acknowledges support from NSERC.

\section{REFERENCES}

1. M. F. Yanik, W. Suh, Z. Wang, and S. Fan, Phys. Rev. Lett. 93, 233903 (2004).

2. J. Scheuer, G. T. Paloczi, J. K. S. Poon, and A. Yariv, Optics \& Photonics News 16, 36-40 (2005).

3. M. Fleischhauer, and M. D. Lukin, Phys. Rev. Lett. 84, 5094-5097 (2000).

4. M. Fleischhauer, A. Imamoglu, and J. P. Marangos, Rev. Mod. Phys. 77, 633-673 (2005).

5. C. Liu, Z. Dutton, C. H. Behroozi, and L. V. Hau, Nature 409, 490-493 (2001).

6. D. F. Phillips, A. Fleischhauer, A. Mair, R. L. Walsworth, and M. D. Lukin, Phys. Rev. Lett. 86, 783-786 (2001).

7. Y. Okawachi, M. Foster, J. Sharping, A. Gaeta, Q. Xu, and M. Lipson, Opt. Express 14, 2317-2322 (2006).

8. Q. Xu, P. Dong, and M. Lipson, Nature Phys. 3, 406-410 (2007).

9. A. Cleland, Nature Phys. 5, 458-460 (2009).

10. M. Eichenfield, J. Chan, R. M. Camacho, K. J. Vahala, and O. Painter, Nature 462, 78-82 (2009).

11. J. D. Joannopoulos, S. G. Johnson, J. N. Winn, and R. D. Meade, Photonic Crystals: Molding the Flow of Light, 2nd ed., Princeton University Press, 2008.

12. M. Maldovan, and E. L. Thomas, Appl. Phys. Lett. 88, 251907 (2006).

13. M. Notomi, E. Kuramochi, and T. Tanabe, Nat. Photonics 2, 741-747 (2008).

14. I. Wilson-Rae, N. Nooshi, W. Zwerger, and T. J. Kippenberg, Phys. Rev. Lett. 99, 093901 (2007).

15. F. Marquardt, J. P. Chen, A. A. Clerk, and S. M. Girvin, Phys. Rev. Lett. 99, 093902 (2007).

16. O. Arcizet, P. Cohadon, T. Briant, M. Pinard, and A. Heidmann, Nature 444, 71-74 (2006).

17. S. Gigan, H. R. Böhm, M. Paternostro, F. Blaser, G. Langer, J. B. Hertzberg, K. C. Schwab, D. Bäuerle, M. Aspelmeyer, and A. Zeilinger, Nature 444, 67-70 (2006).

18. A. Schliesser, P. Del'Haye, N. Nooshi, K. J. Vahala, and T. J. Kippenberg, Phys. Rev. Lett. 97, 243905 (2006).

19. S. Gröblacher, K. Hammerer, M. Vanner, and M. Aspelmeyer, Nature 460, 724-727 (2009).

20. M. F. Yanik, and S. Fan, Phys. Rev. A 71 (2005).

21. E. Figueroa, F. Vewinger, J. Appel, and A. I. Lvovsky, Opt. Lett. 31, 2625-2627 (2006).

22. A. H. Safavi-Naeini, and O. Painter, ArXiv e-prints (2010), 1003.5265.

23. J. Rosenberg, Q. Lin, and O. Painter, Nat. Photonics 3, 478-483 (2009).

24. Q. Lin, J. Rosenberg, D. Chang, R. Camacho, M. Eichenfield, K. J. Vahala, and O. Painter, ArXiv e-prints (2009), 0908.1128. 